Article

\title{
Quality Monitoring for Micro Resistance Spot Welding with Class-Imbalanced Data Based on Anomaly Detection
}

\author{
Jiaquan Zeng, Biao Cao * and Ran Tian \\ School of Mechanical and Automotive Engineering, South China University of Technology, \\ Guangzhou 510640, China; mezengjiaquan@mail.scut.edu.cn (J.Z.); 201821002150@mail.scut.edu.cn (R.T.) \\ * Correspondence: mebcao@scut.edu.cn
}

Received: 18 May 2020; Accepted: 17 June 2020; Published: 19 June 2020

\begin{abstract}
Micro resistance spot welding (MRSW) is an important technology widely used in electronics manufacturing for micro component joining. For the joining of micro enameled wire, quality control is heavily dependent on manual inspection till now. In this paper, a quality monitoring approach based on isolation forest (iForest) is proposed to identify abnormal welds and normal welds. Electrode voltage and welding current of over 110,000 spot welds were collected from a production line. The dynamic resistance and heat input were calculated for all welds and used for feature extraction. A class imbalance problem existed in the collected dataset because abnormal welds were far fewer than normal welds. The anomaly detection model based on iForest was established for the imbalanced data classification after comparison with other methods such as one-class (support vector machine) SVM and local outlier factor. Test results show that the similarity of dynamic resistance profile and heat input compared with the previous ten welds are valid features for detecting a part of the abnormal welds. The iForest model is effective for distinguishing incomplete fusion welds from normal welds with high efficiency. It can assist in the on-line quality monitoring of enameled wire welding process in production.
\end{abstract}

Keywords: micro resistance spot welding; quality monitoring; class-imbalanced data; anomaly detection; isolation forest

\section{Introduction}

With the booming development of miniaturization and integration of electronic devices, micro enameled wires have been increasingly used in the manufacture of various electronic products, such as electroacoustic devices, micro coils, micro delay and chip inductor et al. [1,2]. The micro resistance spot welding (MRSW) of enameled wire to pad is a common production process, since the removal of insulation coating and the joining of wire to pad can be accomplished efficiently in a welding process. Because of advantages such as low cost, high efficiency and ease of automation, MRSW is an important micro joining technology for the manufacture of micro devices, which is also termed small-scale resistance spot welding [3,4].

In view of the demand of reliable signal transmission in electronic components, especially the high fidelity requirement of electroacoustic devices in smartphones and earphones, it is important to ensure the quality of each MRSW weld. However, quality control still relies heavily on manual inspection, which requires experienced people to do it with great attention and still overlooks some defects. It is essential to realize on-line quality monitoring.

Several studies have recently focused on the quality control of MRSW [4-8]. Wan et al. [4] developed a weld quality evaluation model based on electrode voltage recognition for the MRSW 
of 0.4-mm-thick titanium alloy sheets. Wan et al. [5] compared the quality assessment performance between back propagation neural network (BPNN) and probabilistic neural network (PNN) in MRSW, and it was concluded that BPNN was more appropriate for failure load estimation and PNN was more effective at quality classification. Yue et al. [6] established regression equations between welding parameters and weld quality with response surface methodology for foil metal joining. Chen et al. [7] designed multi-performance optimization for MRSW with a hybrid approach. Rikka et al. [8] optimized the welding parameters by Taguchi design to obtain low electrical contact resistance for joining a nickel tab to an inner aluminum casing in a cylindrical cell. However, despite the great demand in industry, there is a lack of investigation into quality monitoring in the MRSW for micro enameled wire joining.

Quality assessment for resistance spot welding (RSW) has been studied extensively $[9,10]$. Different process signals are analyzed for feature extraction, such as dynamic resistance [11], welding power [12], electrode force [13], electrode displacement [14] and multiple signals [15]. Dynamic resistance has wide application because of the low cost and convenient installation of sensors. In recent years, machine learning models have been found to be effective for predicting weld quality, including artificial network [16,17], decision tree [18], random forest [19] and SVM [20]. The rapid development of machine learning technology provides effective decision support tools for weld quality evaluation.

Despite comprehensive studies on the weld quality of MRSW and RSW, specific applications require further investigation. First, compared with RSW or MRSW of metal sheets, MRSW of enameled wire to pad is quite a different physical process and has rarely been explored before. Additionally, a class imbalance problem exists in the quality classification of MRSW in the production. This refers to data within which one or more of the classes (majority class) have a much greater number of samples than the others (minority class) [21]. As a result of quality control, abnormal welds are generally far fewer than normal welds in the manufacture of micro components by MRSW. Traditional classification models cannot provide good performance for class-imbalanced data, which requires imbalanced data processing methods like anomaly detection algorithms [21].

Based on an imbalanced dataset from industry, this work focuses on the quality monitoring for MRSW of micro enameled wire to pad. The welding process was analyzed in terms of dynamic resistance and heat input, and they were adopted for feature extraction. A classification approach based on isolation forest (iForest) is proposed to assist in the quality inspection.

\section{Materials and Methods}

\subsection{Materials and Equipment}

Enameled copper wire with a diameter of $0.08 \mathrm{~mm}$ was joined to the $1.5 \times 1.5 \mathrm{~mm}$ pad coated with $0.01 \mathrm{~mm}$ thick tin solder in an electronics factory. A direct current (DC) welder was used to heat the tungsten electrode, as shown schematically in Figure 1. The joule heat was generated intensively at the electrode tip and transferred to workpieces. The constant voltage control mode was selected for micro enameled wire joining, since it had better adaptability than constant current and constant power, which made the welding current vary in the welding process.

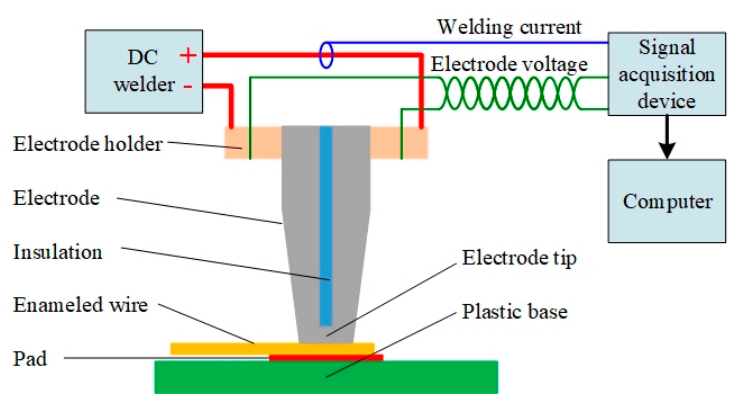

(a)

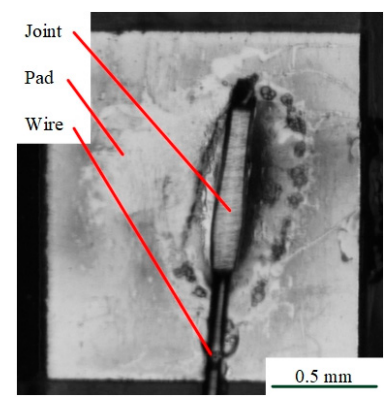

(b)

Figure 1. Cont. 


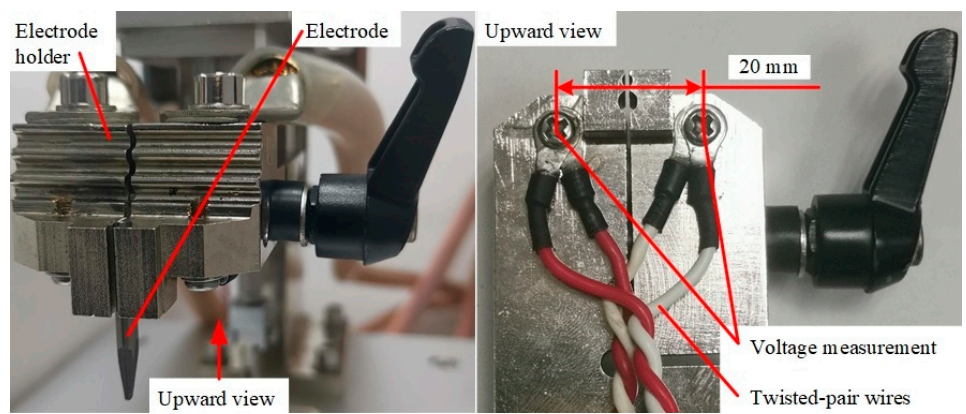

(c)

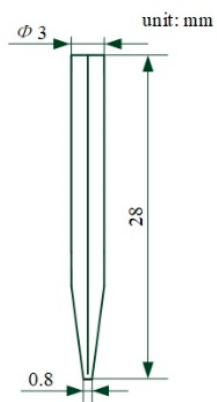

(d)

Figure 1. Representation of MRSW: (a) schematic diagram of experimental setup; (b) a normal weld; (c) electrode and its holder; (d) main dimensions of electrode.

The welding parameters are listed in Table 1 . All welds were produced by applying double heating pulses. The 1st pulse was used to remove the insulation coating from the wire, and the 2nd pulse was set up to provide enough heat. The voltage setting was varied in the range of $0.68-0.90 \mathrm{~V}$, because it matched with each electrode and was adjusted manually during the electrode life. The welding time was varied from 55 to $60 \mathrm{~ms}$ due to its fine-tuning for each electrode.

Table 1. Welding parameters.

\begin{tabular}{cccc}
\hline Number of Heating Pulse & Voltage Setting (V) & Welding Time (ms) & Electrode Force (N) \\
\hline 2 & $0.68-0.90$ & $55-60$ & 5.2 \\
\hline
\end{tabular}

\subsection{Process Data Aacquisition}

The instantaneous electrode voltage $\left(u_{t}\right)$ and welding current $\left(i_{t}\right)$ of 111,958 spot welds were collected by the data acquisition system shown schematically in Figure 1 . The curves of $u_{t}, i_{t}$ and dynamic resistance $\left(R_{t}\right)$ were saved for each weld. A weld performed in the early and middle stage of electrode life is taken as an example in Figure 2. Part of the resistance curve is ignored because $u_{t}$ and $i_{t}$ change rapidly between the two pulses, making the curve change abruptly.

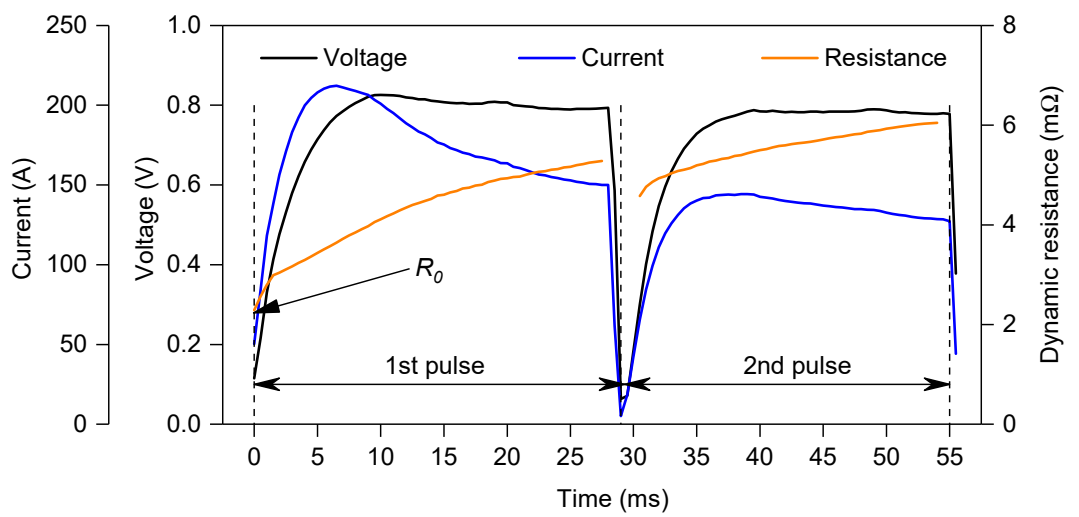

Figure 2. Curves of voltage, current and dynamic resistance collected in a welding process.

\subsection{Weld Quality Determination}

The quality of each weld was estimated by manual visual testing. The quality level was divided into two classes: 'normal' and 'abnormal'. As listed in Table 2, 53 abnormal welds were recorded in detail in the MRSW dataset, including four defect types: incomplete fusion, abnormal wire, overheating, and other failures. 
It is a class-imbalanced dataset, since abnormal welds (the minority class) are far fewer than normal welds (the majority class). Classifying imbalanced data significantly challenges traditional classification models: (1) their bias towards the majority class causes poor identification for the minority class, (2) minority samples usually overlap with majority samples in part of the characteristic space, which is termed class overlap, and (3) there are very few minority samples or even there are only normal samples in specific applications [21,22]. It is more appropriate to select anomaly detection algorithms rather than traditional classification methods for quality classification of imbalanced data.

Despite the low defect rate $(<0.25 \%)$, it is crucial to detect abnormal welds because it is necessary to ensure the quality of each product. They cannot be avoided completely due to various disturbances at production sites. Moreover, it is virtually impossible to make a redundant welding spot on the pad to reduce the failure risk, which makes quality monitoring critical.

Table 2. Visual testing results.

\begin{tabular}{cccccc}
\hline Normal Welds & Incomplete Fusion & Abnormal Wire & Overheating & Other Failures & Defectrate \\
\hline 111,905 & 21 & 22 & 8 & 2 & $<0.25 \%$ \\
\hline
\end{tabular}

\subsection{Welding Process Analysis}

For joining micro enameled wire to tinned pad by MRSW, the basic principle is that the joule heat is generated at the electrode tip by passing an electrical current to remove the insulation coating from the wire and re-flow the solder on the pad. The wire is joined to the pad under a controlled pressure. The weld energy required is obtained from the heated electrode by heat transfer. It is different from the sheet metal joining by RSW or MRSW, where the heat is generated at the interface of the sheets.

Figure 3 shows four signal curves of MRSW from the perspective of electrode life. One of the electrodes is shown as an example: voltage control setting $\left(V_{C O N}\right)$, average of $I_{t}$ in a welding process $\left(I_{A V E}\right)$, initial resistance $\left(R_{0}\right.$, the first resistance value collected in a welding process, shown in Figure 2) and heat input of electrode and workpieces $\left(Q_{E}\right)$. For $I_{A V E}, R_{0}$ and $Q_{E}$, the moving averages of every 200 welds are calculated to make the trends clear. As welding number grows, Figure 3 shows that $V_{C O N}$ is reduced manually (from 0.86 to $0.76 \mathrm{~V}$ ), $I_{A V E}$ and $Q_{E}$ decline in general (from 177 to $88 \mathrm{~A}$, from 8.2 to $3.6 \mathrm{~J}$ ), while the $R_{0}$ curve shows an upward trend (from 2.5 to $8.3 \mathrm{~m} \Omega$ ).

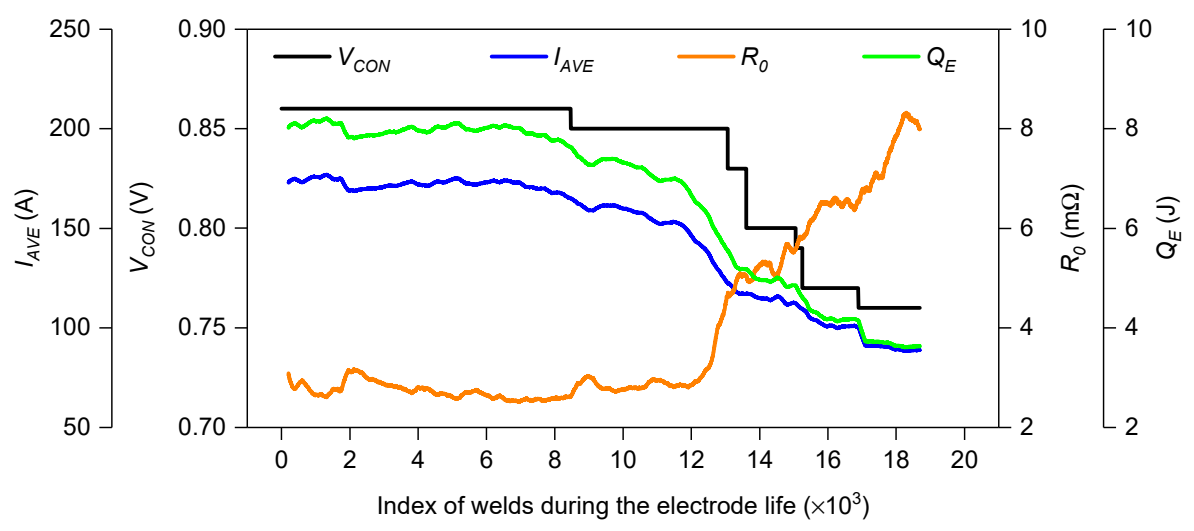

Figure 3. Curves of $V_{C O N}, I_{A V E}, R_{0}$ and $Q_{E}$ during the electrode life.

\subsubsection{Dynamic Resistance}

For the sheet metal joining by RSW in industry, the measured voltage is composed of a resistive and an inductive component: $u_{\mathrm{RSW}}=i R+L d i / d t[23,24]$. The induced voltage is picked up by the two electrical wires which span the upper and lower electrodes and form an inductance loop [24]. However, for the micro enameled wire joining by MRSW with single electrode, there is not an obvious inductance loop for the $u_{t}$ measurement (Figure 1c). The $R_{t}$ is obtained through dividing $u_{t}$ by $i_{t}$ in this study. 
As shown in Figure 4, the equivalent circuit for the electrode and workpieces can be regarded as resistance in series-parallel connection. $R_{E}$ represents the resistance from the voltage measuring position to the electrode tip, $R_{\text {Tip }}$ represents the electrode tip resistance, and $R_{W}$ is used to analyze the workpiece resistance and the contact resistance. The following factors influence $R_{t}$ and its profile.

- Factor 1: the electrode wear. This is caused by several factors, such as frequent tip cleaning and ignition loss, which causes the mass and the size of the tip to decrease (Figure $4 \mathrm{~b}$ ) and $R_{\text {Tip }}$ to increase as the welding number grows [2].

- Factor 2: the temperature increase. The electrode temperature increases because of the net energy input of electrode, and the heat transferred to the workpieces also causes their temperature to increase. Therefore, the resistivity of the electrode and workpieces increases in the welding process.

- Factor 3: the shunting effect. Because of the low resistivity of copper, more or less of the current is diverted to the joint of copper wire to the pad. The $R_{W}$ starts to influence $R_{t}$ after the insulation coating is removed. When the $R_{\text {Tip }}$ becomes higher in the middle and late stage of the electrode life, the resistance curve may drop obviously due to the shunting effect.

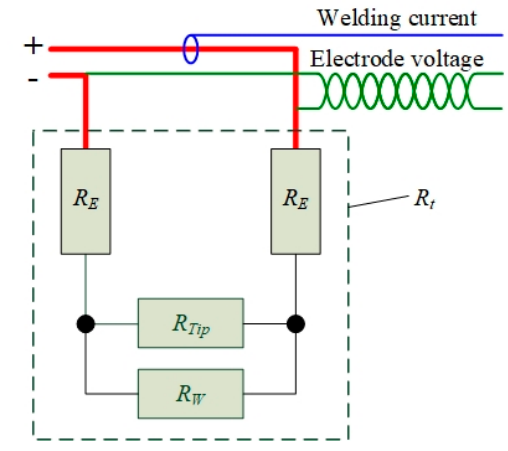

(a)

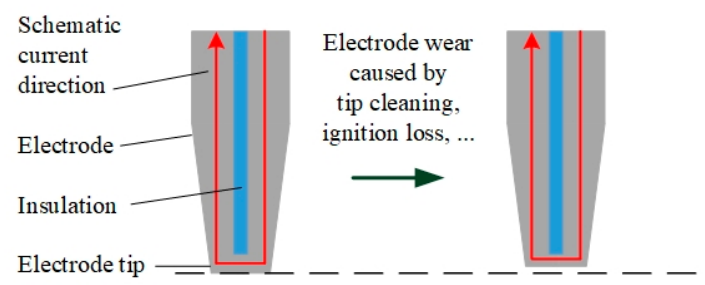

(b)

Figure 4. Schematic diagram for dynamic resistance analysis: (a) equivalent circuit; (b) electrode wear.

According to the change of $R_{t}$ profile during the electrode life, it is divided into several types based on its main trend described in Table 3. One of the electrodes is taken as an example in Figure 5 to show the change of $R_{t}$. Figure 6 shows various profiles with arrows used to point out the main trends. Its change can be explained by the above factors.

1. Factor 1: its effect can be observed from the $R_{0}$, due to the slight effect of Factor 2 and Factor 3 when the welding just starts. After the electrode has been used for a long time, it is prone to wear out, which is reflected in the steep rise of $R_{0}$. It can be observed in Figure 3 also.

2. Factor 2: it generates the 'Up' profile, which is the majority of the resistance curves shown in Figure 5.

3. Factor 3: it can be seen obviously in the middle and late stage of electrode life. After the removal of the insulation coating, part of current is shunted to the wire and the pad, causing $R_{t}$ to drop to some extent. Figure 5 shows the 'Up\&Down' profile, but the 'Down' profile may occur because of the greater effect of Factor 3 than that of Factor 2.

4. The balance between Factor 2 and Factor 3 may result in the 'Flat' profile.

The profile code listed in Table 3 was attributed to each resistance curve by the trend determination rules written in Python. As listed in Table 4, 'Up-Up' and 'Up\&Down-Up' have a majority of $R_{t}$ profiles, accounting for $86.54 \%$ of the total weld count. 
Table 3. Notation of dynamic resistance profile.

\begin{tabular}{cccc}
\hline Main Trend & Pulse & Code & Notation \\
\hline Up & 1 st & 0 & $R_{t}$ keeps rising mainly. \\
Up\&Down & 1 st & 1 & $R_{t}$ starts to rise and then a drop occurs. However, it does not decline in general. \\
Down & 1 st & 2 & $R_{t}$ declines in general since its decline is obviously greater than its rise. \\
Up & 2nd & 0 & $R_{t}$ keeps rising mainly. \\
Flat & 2nd & 1 & $R_{t}$ has obvious stages of slight change despite the local increase. \\
Down & 2nd & 2 & $R_{t}$ declines in general despite the local increase. \\
\hline
\end{tabular}

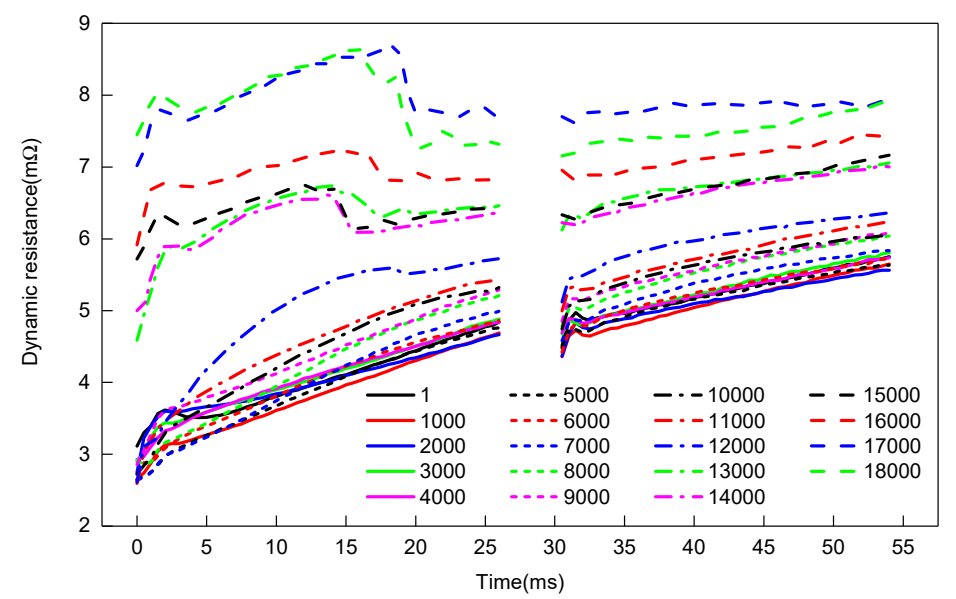

Figure 5. Change of dynamic resistance profile during the electrode life.

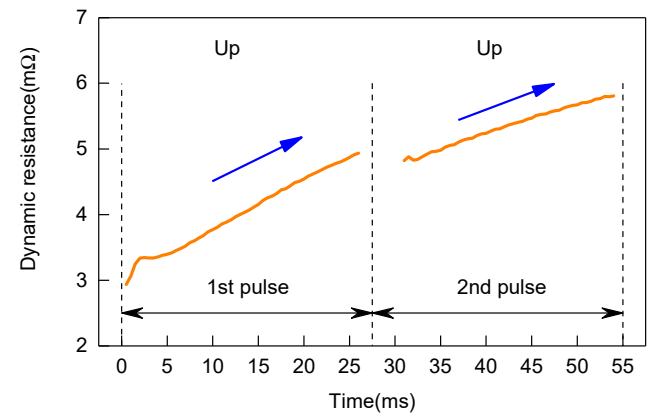

(a)

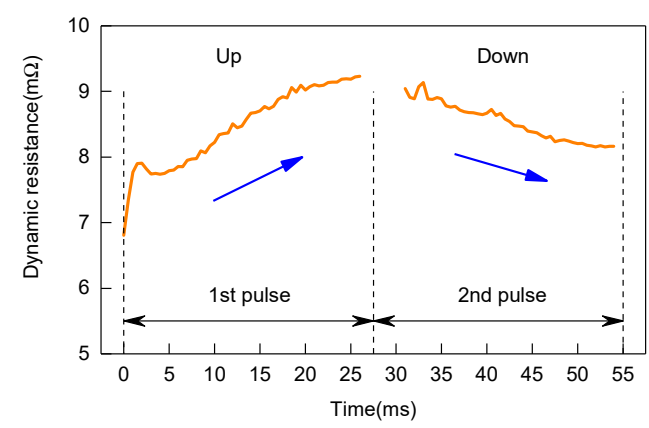

(c)

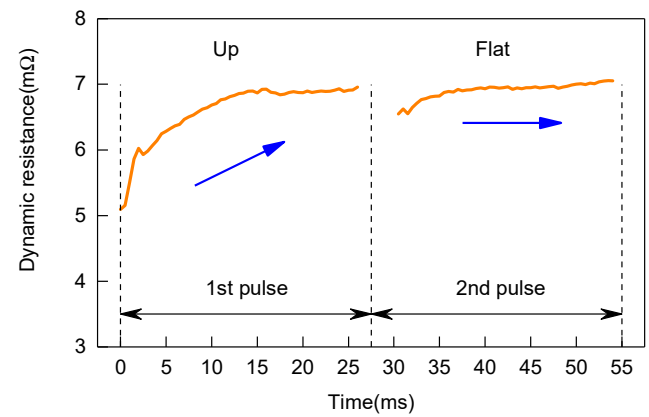

(b)

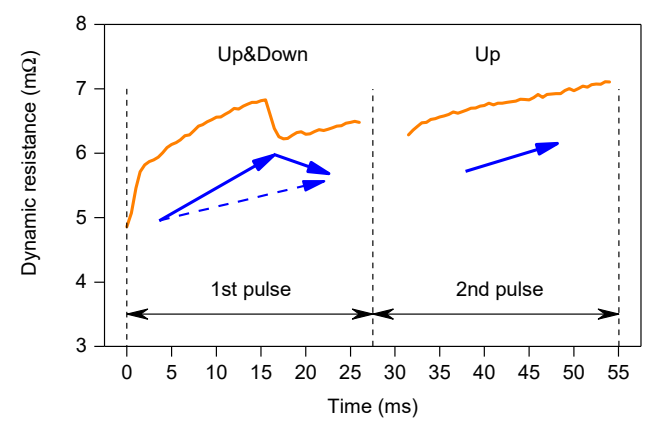

(d)

Figure 6. Cont. 


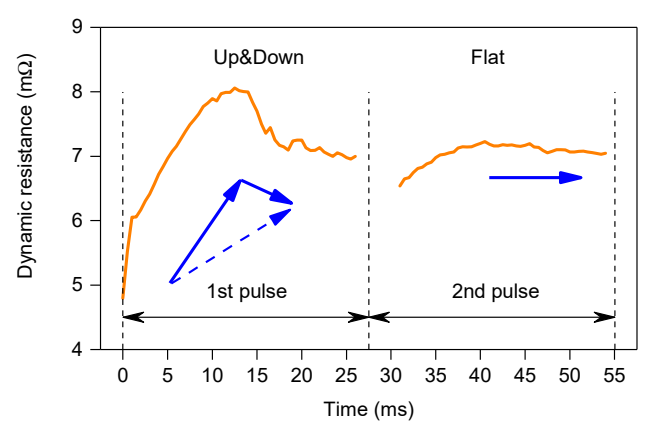

(e)

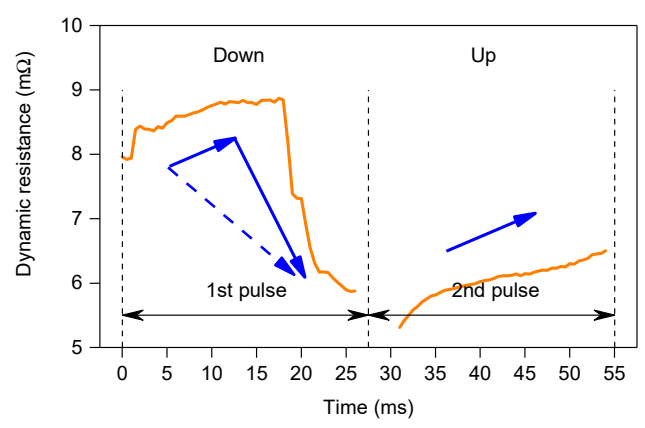

(g)

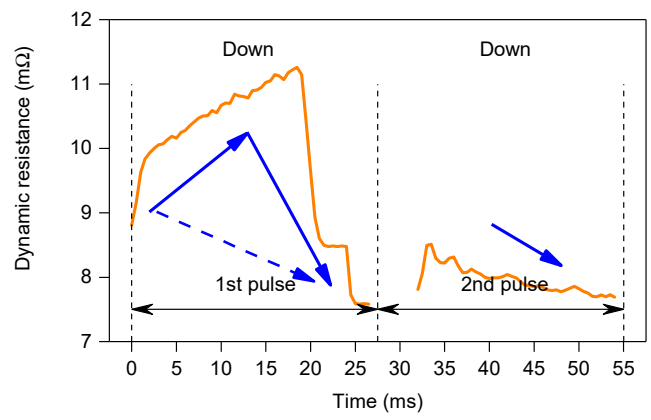

(i)

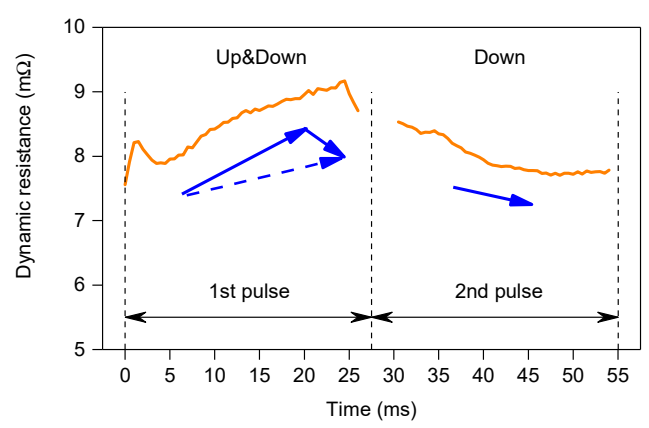

(f)

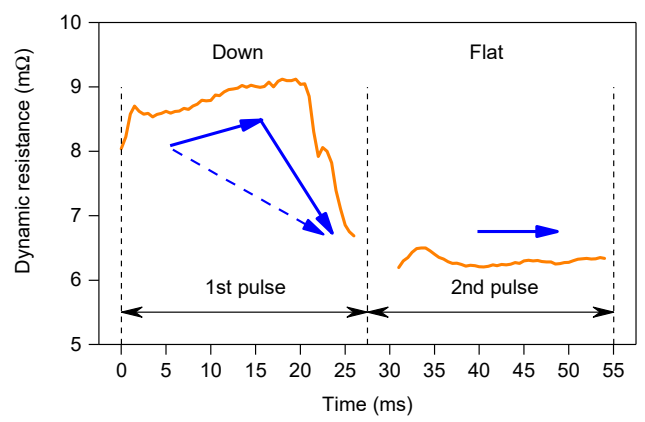

(h)

Figure 6. Dynamic resistance profiles: (a) Up-Up profile; (b) Up-Flat profile; (c) Up-Down profile; (d) Up\&Down-Up profile; (e) Up\&Down-Flat profile; (f) Up\&Down-Down profile; (g) Down-Up profile; (h) Down-Flat profile; (i) Down-Down profile.

Table 4. Ratios of different profile types (\%).

\begin{tabular}{cccccc}
\hline Profile Type & Ratio & Profile Type & Ratio & Profile Type & Ratio \\
\hline Up-Up & 59.68 & Up\&Down-Up & 26.86 & Down-Up & 2.10 \\
Up-Flat & 1.95 & Up\&Down-Flat & 5.88 & Down-Flat & 0.65 \\
Up-Down & 1.73 & Up\&Down-Down & 1.07 & Down-Down & 0.08 \\
\hline
\end{tabular}

\subsubsection{Heat Input}

The $Q_{E}$ is obtained based on the electric work formula $Q_{E}=\int u_{t} i_{t} d t$. As shown in Figure 3, the characteristics of $Q_{E}$ during the electrode life include three aspects. (1) When the $V_{C O N}$ remains unchanged, $Q_{E}$ decreases gradually as the welding number grows due to electrode wear. (2) The electrode is finally scrapped because of insufficient $Q_{E}$. (3) The $V_{C O N}$ is turned down manually during the electrode life. This is related to the following factors: the mass loss of the electrode tip, the heat generated at the tip and the heat transferred to the workpieces. The formula for specific heat capacity $(Q=m c \Delta T)$ can be used for explanation. As welding number grows, the net energy input of the 
electrode decreases from $Q_{1}$ to $Q_{2}$ and its mass decreases from $m_{1}$ to $m_{2}$. However, if $Q_{2} / Q_{1}$ is greater than $m_{2} / m_{1}, \Delta T_{2}$ will be greater than $\Delta T_{1}$, which causes excessive heat transferred to workpieces. Actually, overheating will occur if the $V_{\mathrm{CON}}$ is not duly adjusted in this application.

\subsection{Methods}

\subsubsection{Anomaly Detection Algorithms}

For imbalanced data classification, the pattern of normal samples can be recognized by anomaly detection algorithms when the training set has only normal samples or it contains abnormal samples. The samples that do not fit the pattern are classified as anomalies. This is the basic principle of anomaly detection, which belongs to unsupervised learning. Three well-known anomaly detection algorithms are discussed for quality monitoring: Isolation Forest (iForest), One-Class SVM (OCSVM) and Local Outlier Factor (LOF).

The iForest 'isolates' a sample from the rest of samples by adopting an ensemble of random trees as base estimators for the decision. The number of separations required to isolate a sample is the normality measure of iForest, as it produces noticeably smaller separation numbers for anomalies [25]. OCSVM has good performance for capturing the shape of the training set which contains normal samples only [26]. Samples outside the shape frontier are regarded as anomalies. LOF is a well-known density-based method which identifies the samples with much smaller local density as anomalies [27].

\subsubsection{Feature Extraction}

Similarity is a common measure for distinguishing abnormal samples from normal samples. In terms of $R_{t}$ profile and $Q_{E}$, most welds are similar to their 'near neighbors' (e.g., the previous 10 welds), because the $R_{t}$ and $Q_{E}$ in general change gradually during the electrode life. Compared with the 'far neighbors' (e.g., the previous 1000th-1010th welds), a large disparity may be observed due to electrode wear, especially for welds performed in the middle and late stage of the electrode life.

To quantify the profile similarity between the resistance curves of two welds ( $X$ and $Y$ ), a curve similarity measure $S_{p}(X, Y)$ is constructed using Equations (1)-(3) to obtain the dimensionless feature in the range of [0.0,1.0] [28]. This is based on Euclidean distance, which is widely used to calculate curve similarity [29]. For different cases (profile codes are equal or not), it has different parameter values to calculate the similarity score [30]. When the spot welds have the same profile code in the 1st or 2nd pulse ( $p=1$ or 2), $S_{p}(X, Y)$ is in the range of $[0.0,0.5]$. If not, $S_{p}(X, Y)$ is in the range of $(0.5,1.0]$. $S_{p}(X, Y)$ is smaller when $X$ and $Y$ have more similar profiles.

$$
\begin{gathered}
E_{p}(X, Y)=\sqrt{\sum_{i=1}^{m}\left(x_{i}-y_{i}\right)^{2}} \\
D_{p}(X, Y)=\max \left(\frac{1}{m} \sum_{i=1}^{m} x_{i}, \frac{1}{m} \sum_{i=1}^{m} y_{i}, E_{p}(X, Y)\right) \\
S_{p}(X, Y)=\frac{1}{2}\left(A+\frac{E_{p}(X, Y)}{D_{p}(X, Y)}\right), A= \begin{cases}0 & , C_{p}(X)=C_{p}(Y) \\
1 & , C_{p}(X) \neq C_{p}(Y)\end{cases}
\end{gathered}
$$

where $p$ represents the heating pulse $(p=1,2), m$ is the number of $R_{t}$ collected during the pulse, $E_{p}(X, Y)$ is the Euclidean distance of $X$ and $Y$ in the 1st or 2nd pulse, $D_{p}(X, Y)$ is the maximum of $E_{p}(X, Y)$ and the averages of $X$ and $Y, C_{p}$ represents the profile code, and $S_{p}(X, Y)$ is used to evaluate the profile similarity.

Two features calculated by Equations (4)-(7) are extracted from $Q_{E}$ and $R_{t}$ for anomaly detection: the similarity of $Q_{E}\left(\Delta Q_{10}\right)$ and the similarity of $R_{t}$ profile $\left(\Delta S_{10}\right)$. Both of them are compared with 
previous 10 welds. There is no evident linear correlation between them because the absolute value of Pearson correlation coefficient is less than 0.12 .

$$
\begin{gathered}
\Delta Q_{10}=\frac{Q_{a n l z}-Q_{a v e}}{Q_{a v e}}, Q_{a v e}=\frac{1}{10} \sum_{i=1}^{10} Q_{i} \\
\Delta S_{1-10}=\frac{1}{10} \sum_{i=1}^{10} S_{1}\left(X_{\text {anlz }}, X_{i}\right) \\
\Delta S_{2-10}=\frac{1}{10} \sum_{i=1}^{10} S_{2}\left(X_{\text {anlz }}, X_{i}\right) \\
\Delta S_{10}=\sqrt{0.5 \times\left(\Delta S_{1-10}^{2}+\Delta S_{2-10}^{2}\right)}
\end{gathered}
$$

where symbol anlz represents the weld to be analyzed, $i$ is the index of its previous welds, $\Delta Q_{10}$ is the relative change of $Q_{E}$, and $\Delta S_{10}$ is used to analyze the similarity of $R_{t}$ profile in the double-pulse welding process. For the first two welds of each electrode, the $\Delta Q_{10}$ and $\Delta S_{10}$ are regarded as 0.0; for the 3rd to 10th welds, the nominal $\Delta Q_{10}$ and $\Delta S_{10}$ are compared with the previous two welds.

\subsubsection{Model Construction}

To conduct the anomaly detection experiments, the models are constructed with the training set including normal welds only. All the abnormal welds and the remaining normal welds are used for model verification, as shown in Figure 7. Each weld in the test set is classified as 'normal' or 'abnormal'. The Python packages from scikit-learn [31] are used, and the major parameters are listed in Table 5.

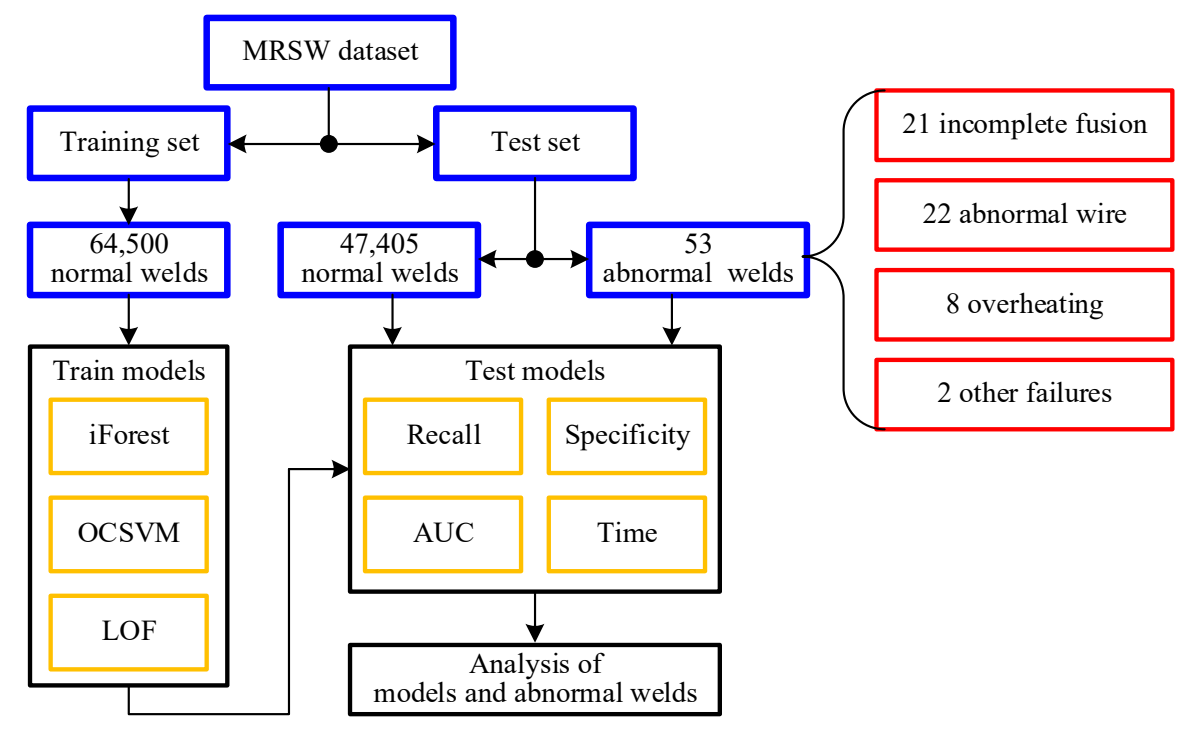

Figure 7. Model construction process.

Four performance measures are applied in this study: recall (also termed sensitivity), specificity, processing time, and the area under the receiver operating characteristic curve (AUC) [20]. Recall and specificity are calculated by Equations (8)-(9). True positive (TP), false positive (FP), true negative (TN) and false negative (FN) are four types of predicted results in binary classification. In general, minority class (abnormal weld) is regarded as positive case and majority class (normal weld) is termed 
as negative case. AUC is widely used to analyze the performance of a binary classifier. It is in the range $[0,1]$, and a good classifier has an AUC score near 1.

$$
\begin{gathered}
\text { Recall }=\frac{T P}{T P+F N} \\
\text { Specificity }=\frac{T N}{T N+F P}
\end{gathered}
$$

As common for preprocessing in machine learning, the features $\Delta Q_{10}$ and $\Delta S_{10}$ are scaled into the interval of $[0,1]$ by normalization. The normalized value $\Delta Q_{10 \mathrm{~N}}$ is calculated by Equation (10), and $\Delta S_{10 \mathrm{~N}}$ is equal to $\Delta S_{10}$, where $\operatorname{Lim}_{\mathrm{L}}$ is the lower limit and $\operatorname{Lim}_{\mathrm{U}}$ is the upper limit used for $\Delta Q_{10 \mathrm{~N}}$. Compared with the previous 10 welds, the welds with small changes in $Q_{E}$ have $\Delta Q_{10 \mathrm{~N}}$ near 0.5 , and $\Delta S_{10 \mathrm{~N}}$ near 0 reflects small change in the $R_{t}$ profile.

$$
\Delta Q_{10 \mathrm{~N}}=\left\{\begin{array}{cc}
0 & , \Delta Q_{10} \leq \operatorname{Lim}_{\mathrm{L}} \\
\frac{\Delta Q_{10}-\operatorname{Lim}_{\mathrm{L}}}{\operatorname{Lim}_{\mathrm{U}}-\operatorname{Lim}_{\mathrm{L}}} & , \Delta Q_{10} \in\left(\operatorname{Lim}_{\mathrm{L}}, \operatorname{Lim}_{\mathrm{U}}\right) \\
1 & , \Delta Q_{10} \geq \operatorname{Lim}_{\mathrm{U}}
\end{array}\right.
$$

Table 5. Anomaly detection algorithms and their major parameters.

\begin{tabular}{cr}
\hline Algorithm & Major Parameters \\
\hline iForest & trees $=100$, subsampling size $=256$, contamination $(c) \in[0.01,0.02, \ldots, 0.49,0.49999]$ \\
OCSVM & $\begin{array}{c}\text { kernel }=\text { 'rbf', gamma }=\text { 'scale', } n u \in[0.01,0.02, \ldots, 0.49,0.49999] \\
\text { LOF }\end{array}$ \\
neighbors $=20$, contamination $(c) \in[0.01,0.02, \ldots, 0.49,0.49999]$ \\
\hline
\end{tabular}

\section{Results and Discussion}

The particularity of the quality classification in this study can be illustrated by the feature distribution of normal welds and abnormal welds shown in Figure 8. The $\operatorname{Lim}_{\mathrm{L}}$ and $\operatorname{Lim}_{\mathrm{U}}$ of $\Delta Q_{10}$ are selected to -0.1 and 0.1 , respectively, because $97.5 \%$ welds are at this interval. According to the statistical analysis, 52,291 welds meet the conditions of $\Delta Q_{10 \mathrm{~N}} \in[0.4,0.6)$ and $\Delta S_{10 \mathrm{~N}} \in[0.0,0.2)$, and $79.6 \%(89,094)$ welds meet the conditions of $\Delta Q_{10 N} \in[0.3,0.7)$ and $\Delta S_{10 N} \in[0.0,0.5)$. This reflects the fact that most welds are similar to their 'near neighbors' in terms of $Q_{E}$ and $R_{t}$ profile.

It can be observed that in this imbalanced dataset, abnormal welds overlap with normal welds in part of the two-dimensional characteristic space. The combination of class imbalance and class overlap makes it difficult to construct the frontier of each class, which adds complexity for quality classification. The dataset of normal welds may contain certain abnormal welds because of misjudgment, which may make abnormal welds overlap 'normal' (misjudged) welds. However, the amount of such polluted data was limited, since the weld quality was inspected by two inspectors in this experiment.

The weld failure detection methods based on traditional classification models appear to be unsuitable to detect abnormal welds from the imbalanced data. First, such models generally adopt global performance measures such as prediction accuracy in the learning process. If all welds are predicted as normal welds, a high accuracy score $(>99.75 \%$ ) can be obtained because of the low defect rate $(<0.25 \%)$, leading to poor detection of weld failure in this application. Second, traditional classification models are based on supervised learning for the class-balanced data in general, while there is not enough real data of weld failure available for model training and testing in this study, since the abnormal welds do not often occur in reality. Third, the problem of class overlap also makes it difficult to distinguish between normal welds and abnormal welds by traditional classification models. 


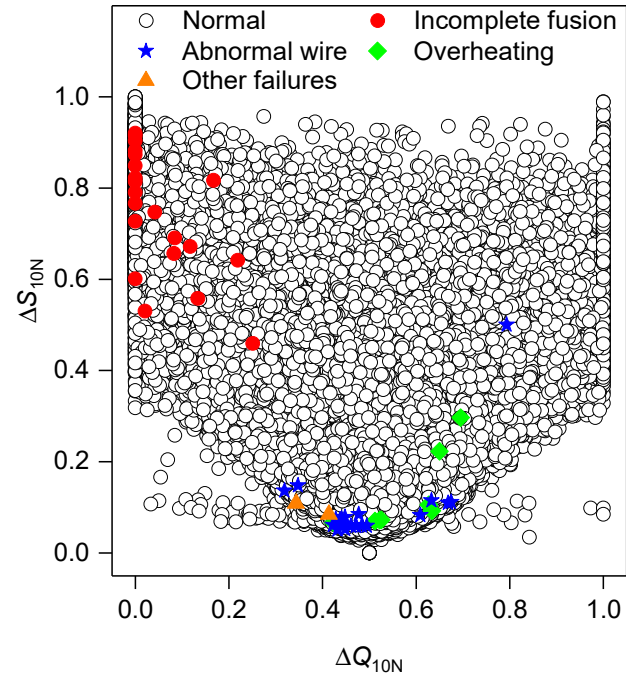

(a)

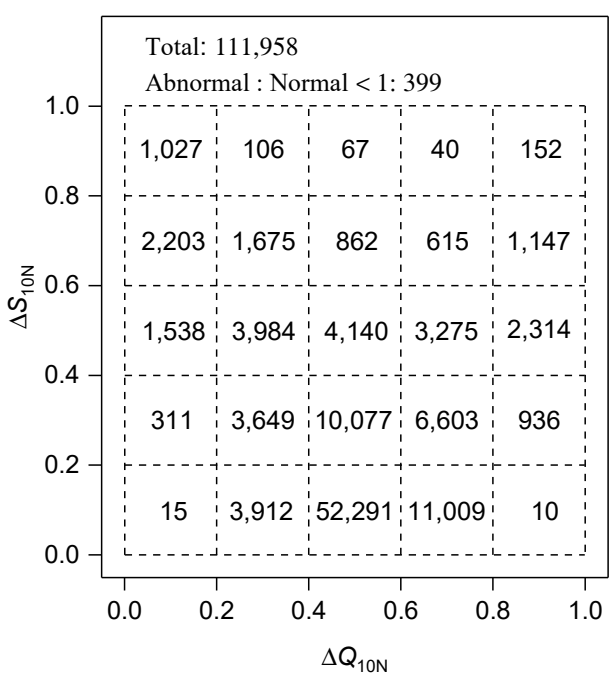

(b)

Figure 8. Feature distribution of normal welds and abnormal welds: (a) characteristic space; (b) frequency distribution.

It is an essential task to find out abnormal welds in the quality monitoring of MRSW. Figure 9 shows the performance difference of anomaly detection for three models and four defect types. The parameter $c$ or $n u$ provided in the packages is used to adjust the model decision function.

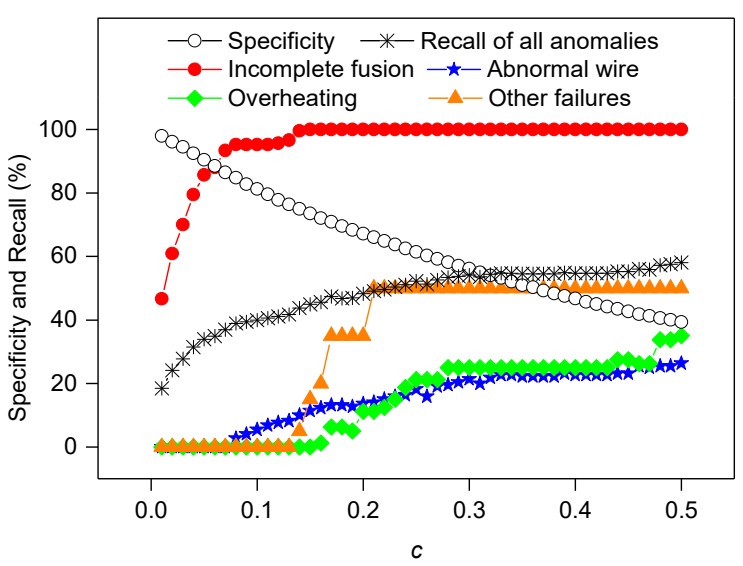

(a)

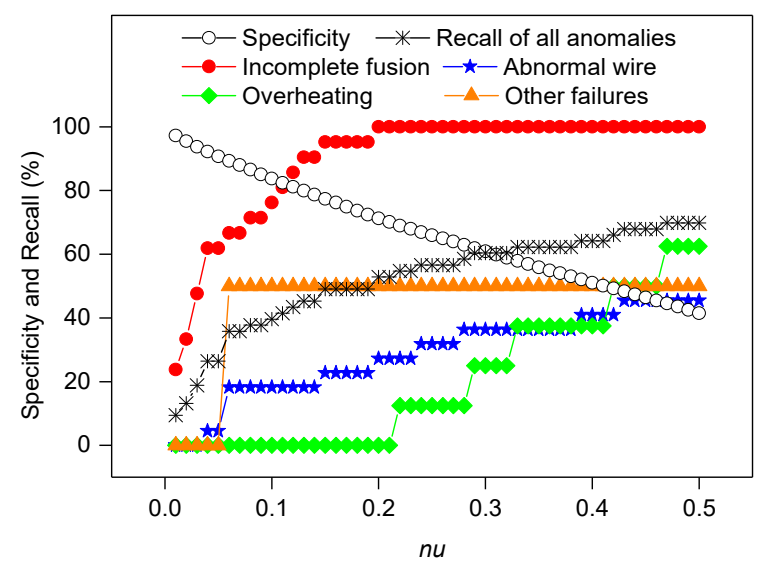

(b)

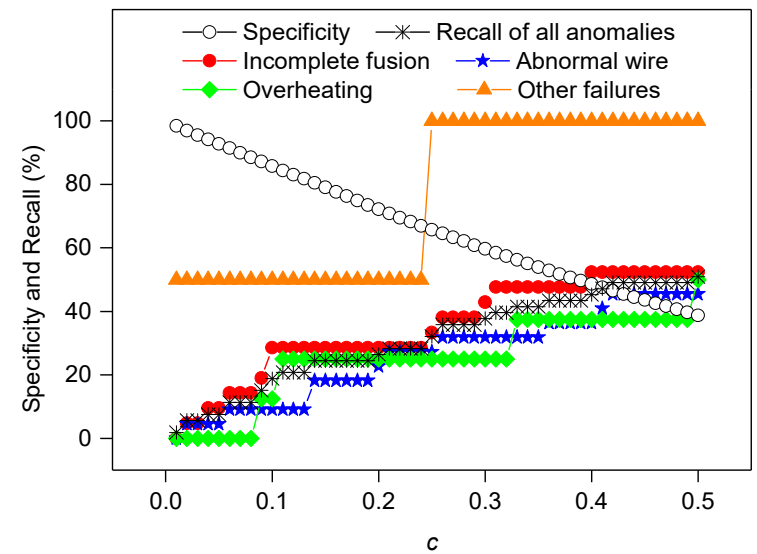

(c)

Figure 9. Comparison of detection performance: (a) iForest, (b) OCSVM; (c) LOF. 
For the detection of incomplete fusion welds, both iForest and OCSVM can distinguish all the incomplete fusion welds from normal welds, at the cost of the decrease in the specificity. The LOF model appears to be unsuitable for this application because its recall of incomplete fusion welds cannot reach $100 \%$ when $c$ is near its upper limit 0.5 . For the detection of other defect types, none of these models can identify the abnormal welds effectively, since no valid features have been extracted from $u_{t}$, $i_{t}$ and variables calculated from them so far.

To analyze the classification of incomplete fusion welds and normal welds further, AUC, specificity and processing time are adopted, as listed in Table 6. The average training time and average test time over the range of $c$ or $n u$ are used to compare the model efficiency. The LOF model is time-saving for model construction in this application, but it cannot identify all the incomplete fusion welds. Hence, its AUC is regarded as NA. The performance of OCSVM for anomaly detection is not poor; however, it is relatively time-consuming to train and test the model. The identification of incomplete fusion welds and normal welds can be performed effectively and efficiently by the iForest model. It has a high AUC score of 0.9525 , and it takes $1.79 \mathrm{~s}$ to train the model with over 60,000 welds in the training set, and $1.11 \mathrm{~s}$ to test over 47,000 welds.

Table 6. AUC, recall and processing time.

\begin{tabular}{cccccc}
\hline \multirow{2}{*}{ Algorithm } & \multirow{2}{*}{ AUC } & \multicolumn{2}{c}{ Recall } & \multicolumn{2}{c}{ Processing Time (s) } \\
\cline { 3 - 6 } & & Specificity of $\mathbf{9 0} \%$ & Specificity of $\mathbf{8 0} \%$ & Train & Test \\
\hline iForest & 0.9525 & 0.8633 & 0.9524 & 1.79 & 1.11 \\
OCSVM & 0.9047 & 0.6437 & 0.9037 & 53.29 & 9.46 \\
LOF & NA & 0.1429 & 0.2857 & 0.43 & 0.25 \\
\hline
\end{tabular}

In addition to identifying abnormal welds, the interpretation of anomalies is also important for quality monitoring. For incomplete fusion welds, one of them and its previous 10 welds are shown as an example in Figure $10 \mathrm{a}, \mathrm{c}, \mathrm{d}$. It can be observed that this abnormal weld has a different $R_{t}$ profile and smaller $Q_{E}$. The previous welds have an 'Up\&Down' profile in the 1st pulse, while the abnormal weld has an 'Up-Down' profile in the double pulses. This implies that the insulation coating was not properly removed as designed in the 1st pulse for this abnormal weld. Since the welding time was fixed, this weld lacked enough energy to join the wire to the pad after the insulation coating was removed. Based on the assumption that normal welds are 'many and similar' and anomalies are 'few and different', the iForest model can detect incomplete fusion welds with good performance in this application.

For other defect types, one from abnormal wire welds is analyzed as an example (Figure 10b). It was caused by the deviation of wire position. As shown in Figure 10e,f, it overlaps with its previous 10 welds in terms of $R_{t}$ profile. This is related to the characteristics of the MRSW process for micro enameled wire joining, implying that the case of abnormal wire which occurs in the early and middle stage of electrode life does not obviously influence the temperature rise of electrode tip and tinned pad. Therefore, there is no remarkable difference in either $\Delta Q_{10 \mathrm{~N}}$ or $\Delta S_{10 \mathrm{~N}}$ between normal welds and these abnormal welds, meaning that the models cannot detect them effectively.

Although only incomplete fusion can be detected by the present iForest model with good performance, it is favorable for the quality monitoring research for MRSW. Its detection requires inspectors to be experienced and focused, which makes it relatively difficult to perform visual testing in the production line, while the deviation of wire position can be identified as abnormal wire with relative ease by a machine vision system. To further separate various abnormal welds from normal welds, more features from other process signals are required to fully represent the welding process. Similar to RSW, the quality monitoring based on multiple signals may be better for MRSW than that based on electrical signals only. Electrode displacement, dynamic force and images before and after welding are expected to be added in future work to improve quality monitoring. 


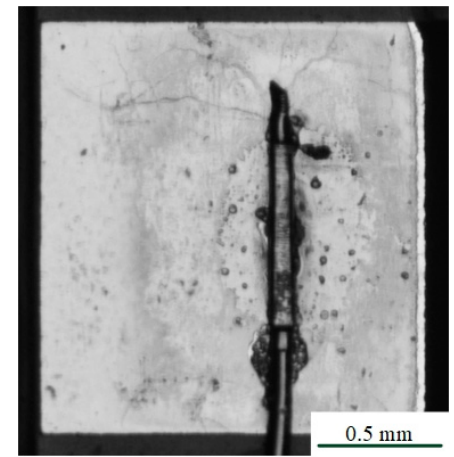

(a)

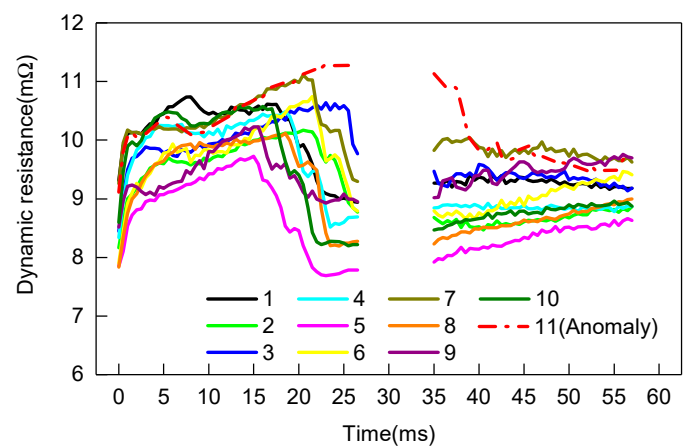

(c)

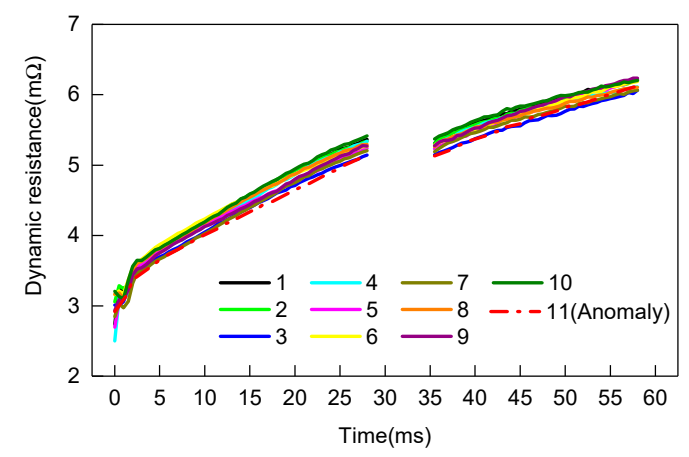

(e)

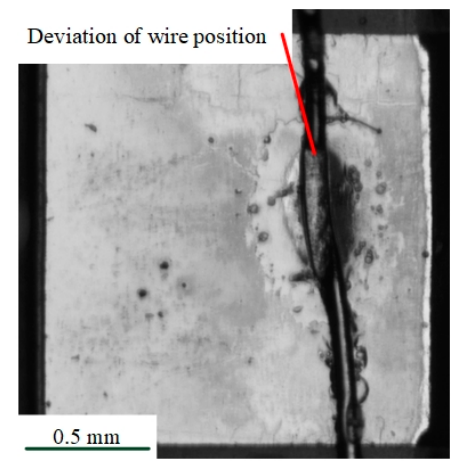

(b)

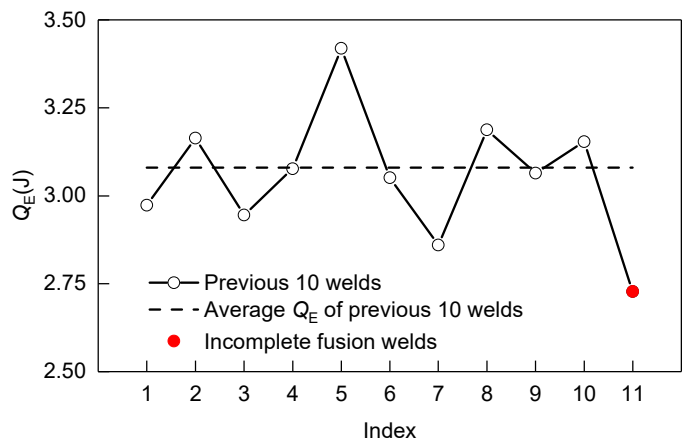

(d)

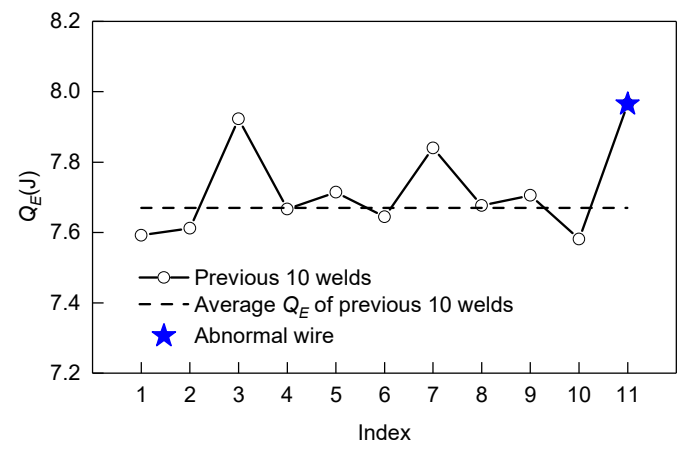

(f)

Figure 10. Abnormal welds and the previous 10 welds: (a) incomplete fusion weld; (b) abnormal wire weld; for incomplete fusion weld: (c) $R_{t}$ profile; (d) $Q_{E}$; for abnormal wire weld: (e) $R_{t}$ profile; (f) $Q_{E}$.

\section{Conclusions}

An anomaly detection model based on iForest is proposed to assist in the quality monitoring of MRSW. The work is based on a dataset with the class imbalance problem. In terms of the dynamic resistance and heat input, the change during the electrode life is analyzed, and features are extracted from them for anomaly detection. The following conclusions can be obtained.

1. Class imbalance and overlap exist in the quality estimation of MRSW production and require proper anomaly detection algorithms for quality monitoring.

2. The similarity of dynamic resistance profile and heat input compared with the previous ten welds are valid features for detecting incomplete fusion welds.

3. For the classification of incomplete fusion welds and normal welds, the iForest model is a good candidate with a high AUC score of 0.9525 and high efficiency. 
Author Contributions: Conceptualization, B.C.; methodology, B.C.; formal analysis, J.Z. and R.T.; investigation, J.Z. and R.T.; resources, B.C.; writing-original draft preparation, J.Z.; writing-review and editing, B.C.; supervision, B.C.; project administration, B.C. All authors have read and agreed to the published version of the manuscript.

Funding: This research received no external funding.

Acknowledgments: The authors would like to thank Kai Yang for technical support of data acquisition system.

Conflicts of Interest: The authors declare no conflict of interest.

\section{References}

1. Yang, S.; Li, Y.; Lin, J. Stripping weld-A new technique of welding enamelled wires by resistance weld. Trans. China Weld. Inst. 2008, 29, 21-25.

2. Zeng, J.; Cao, B.; Yang, K.; Wu, M. Analysis method of electrode ignition loss state in enameled wire resistance spot welding. J. South China Univ. Technol. 2019, 47, 32-37.

3. Zhao, D.; Wang, Y.; Liang, D. Correlating variations in the dynamic power signature to nugget diameter in resistance spot welding using Kriging model. Measurement 2019, 135, 6-12. [CrossRef]

4. Wan, X.; Wang, Y.; Zhao, D. Quality evaluation in small-scale resistance spot welding by electrode voltage recognition. Sci. Technol. Weld. Join. 2016, 21, 358-365. [CrossRef]

5. Wan, X.; Wang, Y.; Zhao, D.; Huang, Y. A comparison of two types of neural network for weld quality prediction in small scale resistance spot welding. Mech. Syst. Signal. Process. 2017, 93, 634-644. [CrossRef]

6. Yue, X.; Tong, G.; Chen, F.; Ma, X.; Gao, X. Optimal welding parameters for small-scale resistance spot welding with response surface methodology. Sci. Technol. Weld. Join. 2017, 22, 143-149. [CrossRef]

7. Chen, F.; Wang, Y.; Sun, S.; Ma, Z.; Huang, X. Multi-objective optimization of mechanical quality and stability during micro resistance spot welding. Int. J. Adv. Manuf. Technol. 2019, 101, 1903-1913. [CrossRef]

8. Rikka, V.R.; Sahu, S.R.; Roy, A.; Jana, S.N.; Sivaprahasam, D.; Prakash, R. Tailoring micro resistance spot welding parameters for joining nickel tab to inner aluminium casing in a cylindrical lithium ion cell and its influence on the electrochemical performance. J. Manuf. Process. 2020, 49, 463-471. [CrossRef]

9. Zhou, K.; Yao, P. Overview of recent advances of process analysis and quality control in resistance spot welding. Mech. Syst. Signal Process. 2019, 124, 170-198. [CrossRef]

10. Xia, Y.; Li, Y.; Lou, M.; Lei, H. Recent advances and analysis of quality monitoring and control technologies for RSW. China Mech. Eng. 2020, 31, 100-125.

11. Adams, D.W.; Summerville, C.; Voss, B.M.; Jeswiet, J.; Doolan, M.C. Correlating variations in the dynamic resistance signature to weld strength in resistance spot welding using principal component analysis. J. Manuf. Sci. Eng. 2017, 139, 44502. [CrossRef]

12. Wang, X.; Zhou, J.; Yan, H.; Pang, C. Quality monitoring of spot welding with advanced signal processing and data-driven techniques. Trans. Inst. Meas. Control 2018, 40, 2291-2302. [CrossRef]

13. Chen, S.; Wu, N.; Xiao, J.; Li, T.; Lu, Z. Expulsion identification in resistance spot welding by electrode force sensing based on wavelet decomposition with multi-Indexes and BP neural networks. Appl. Sci. 2019, 9, 4028. [CrossRef]

14. Xing, B.; Xiao, Y.; Qin, Q. Characteristics of shunting effect in resistance spot welding in mild steel based on electrode displacement. Measurement 2018, 115, 233-242. [CrossRef]

15. Wen, J.; Jia, H.; Wang, C. Quality Estimation system for resistance spot welding of stainless steel. ISIJ Int. 2019, 59, 2073-2076. [CrossRef]

16. Gavidel, S.Z.; Lu, S.; Rickli, J.L. Performance analysis and comparison of machine learning algorithms for predicting nugget width of resistance spot welding joints. Int. J. Adv. Manuf. Technol. 2019, 105, 3779-3796. [CrossRef]

17. Amiri, N.; Farrahi, G.H.; Kashyzadeh, K.R.; Chizari, M. Applications of ultrasonic testing and machine learning methods to predict the static \& fatigue behavior of spot-welded joints. J. Manuf. Process. 2020, $52,26-34$.

18. Boersch, I.; Füssel, U.; Gresch, C.; Großmann, C.; Hoffmann, B. Data mining in resistance spot welding. Int. J. Adv. Manuf. Technol. 2018, 99, 1085-1099. [CrossRef]

19. Xing, B.; Xiao, Y.; Qin, Q.; Cui, H. Quality assessment of resistance spot welding process based on dynamic resistance signal and random forest based. Int. J. Adv. Manuf. Technol. 2018, 94, 327-339. [CrossRef] 
20. Pereda, M.; Santos, J.I.; Martín, O.; Galán, J.M. Direct quality prediction in resistance spot welding process: Sensitivity, specificity and predictive accuracy comparative analysis. Sci. Technol. Weld. Join. 2015, 20, 679-685. [CrossRef]

21. Guo, H.; Li, Y.; Shang, J.; Gu, M.; Huang, Y.; Gong, B. Learning from class-imbalanced data: Review of methods and applications. Expert Syst. Appl. 2017, 73, 220-239.

22. Liu, J.; Gu, J.; Li, H.; Carlson, K.H. Machine learning and transport simulations for groundwater anomaly detection. J. Comput. Appl. Math. 2020, 380, 112982. [CrossRef]

23. Su, Z.; Xia, Y.; Shen, Y.; Li, Y. A novel real-time measurement method for dynamic resistance signal in medium-frequency DC resistance spot welding. Meas. Sci. Technol. 2020, 31, 55011. [CrossRef]

24. Li, W.; Cerjanec, D.; Grzadzinski, G.A. A comparative study of single-phase AC and multiphase DC resistance spot welding. J. Manuf. Sci. Eng. 2005, 127, 583-589. [CrossRef]

25. Liu, F.; Ting, K.; Zhou, Z. Isolation-based anomaly detection. ACM Trans. Knowl. Discov. Data 2012, 6, 1-39. [CrossRef]

26. Schölkopf, B.; Platt, J.; Shawe-Taylor, J.; Smola, A.; Williamson, R. Estimating the Support of a high-dimensional distribution. Neural Comput. 2001, 13, 1443-1471. [CrossRef]

27. Breunig, M.; Kriegel, H.; Ng, R.; Sander, J. LOF: Identifying density-based local outliers. In Proceedings of the 2000 ACM SIGMOD-International Conference on Management of Data, Dallas, Texas, USA, 16-18 May 2000; pp. 93-104.

28. An, H.; Wang, Z.; Wang, G.; Song, Q.; Liu, F.; Zhong, C. Research on on-line monitoring of surface roughness in composite drilling and adaptive optimization of parameters. J. Mech. Eng. 2020, 56, 27-34.

29. Shi, L.; Zhou, R.; Li, J.; Wang, L.; Xu, F.; Wang, Y. New energy-Load characteristic index based on time series similarity measurement. Electr. Pow. Autom. EQ. 2019, 39, 75-81.

30. Qiu, Y.; Hu, H.; Zheng, J.; Chen, Q. On-line handwriting signature verification based on curve similarity. Syst. Eng. Electron. 2014, 36, 1016-1020.

31. Pedregosa, F.; Varoquaux, G.; Michel, V.; Thirion, B.; Grisel, O.; Blondel, M. Scikit-learn: Machine learning in python. J. Mach. Learn. Res. 2011, 12, 2825-2830.

(C) 2020 by the authors. Licensee MDPI, Basel, Switzerland. This article is an open access article distributed under the terms and conditions of the Creative Commons Attribution (CC BY) license (http://creativecommons.org/licenses/by/4.0/). 